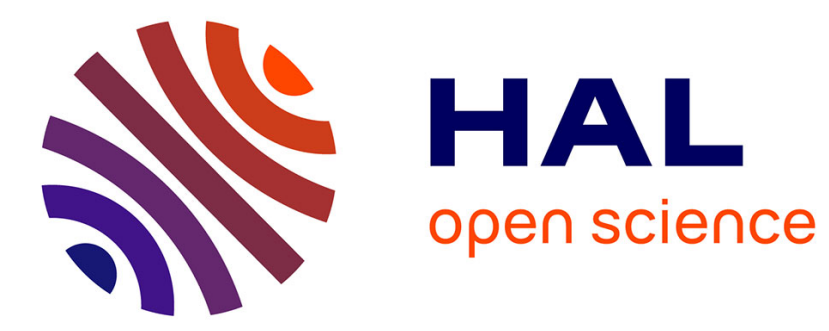

\title{
La Mauritanie en noir et blanc. Petite promenade linguistique en hassâniyya \\ Catherine Taine-Cheikh
}

\section{To cite this version:}

Catherine Taine-Cheikh. La Mauritanie en noir et blanc. Petite promenade linguistique en hassâniyya. Revue des Mondes Musulmans et de la Méditerranée, 1989, 54 (4), pp.90-105. halshs-00456263

\section{HAL Id: halshs-00456263 \\ https://shs.hal.science/halshs-00456263}

Submitted on 13 Feb 2010

HAL is a multi-disciplinary open access archive for the deposit and dissemination of scientific research documents, whether they are published or not. The documents may come from teaching and research institutions in France or abroad, or from public or private research centers.
L'archive ouverte pluridisciplinaire HAL, est destinée au dépôt et à la diffusion de documents scientifiques de niveau recherche, publiés ou non, émanant des établissements d'enseignement et de recherche français ou étrangers, des laboratoires publics ou privés. 


\section{Persée}

http://www.persee.fr

\section{La Mauritanie en noir et blanc. Petite promenade linguistique en hassâniyya}

\section{Catherine Taine-Cheikh}

Revue du monde musulman et de la Méditerranée, Année 1989, Volume 54, Numéro 1

p. $90-105$

Voir l'article en ligne

Avertissement L'éditeur du site « PERSEE » - le Ministère de la jeunesse, de l'éducation nationale et de la recherche, Direction de l'enseignement supérieur, Sous-direction des bibliothèques et de la documentation - détient la propriété intellectuelle et les droits d'exploitation. A ce titre il est titulaire des droits d'auteur et du droit sui generis du producteur de bases de données sur ce site conformément à la loi n98-536 du 1er juillet 1998 relative aux bases de données.

Les oeuvres reproduites sur le site «PERSEE » sont protégées par les dispositions générales du Code de la propriété intellectuelle.

Droits et devoirs des utilisateurs

Pour un usage strictement privé, la simple reproduction du contenu de ce site est libre. Pour un usage scientifique ou pédagogique, à des fins de recherches, d'enseignement ou de communication excluant toute exploitation commerciale, la reproduction et la communication au public du contenu de ce site sont autorisées, sous réserve que celles-ci servent d'illustration, ne soient pas substantielles et ne soient pas expressément limitées (plans ou photographies). La mention Le Ministère de la jeunesse, de l'éducation nationale et de la recherche, Direction de l'enseignement supérieur, Sous-direction des bibliothèques et de la documentation sur chaque reproduction tirée du site est obligatoire ainsi que le nom de la revue et- lorsqu'ils sont indiqués - le nom de l'auteur et la référence du document reproduit.

Toute autre reproduction ou communication au public, intégrale ou substantielle du contenu de ce site, par quelque procédé que ce soit, de l'éditeur original de l'oeuvre, de l'auteur et de ses ayants droit.

La reproduction et l'exploitation des photographies et des plans, y compris à des fins commerciales, doivent être autorisés par l'éditeur du site, Le Ministère de la jeunesse, de l'éducation nationale et de la recherche, Direction de l'enseignement supérieur, Sous-direction des bibliothèques et de la documentation (voir http://www.sup.adc.education.fr/bib/ ). La source et les crédits devront toujours être mentionnés. 


\section{LA MAURITANIE EN NOIR ET BLANC Petite promenade linguistique en hassāniyya}

En général on considère les langues d'un point de vue essentiellement utilitaire. On attend d'elles un comportement servile de bon outil de communication et on aura pour les dialectes (ces "sous-langues" immatures) une attitude particulièrement condescendante, sachant qu'ils risquent de vous trahir au détour d'une conversation. Si d'aventure on se prend à les interroger sérieusement, c'est pour compter et classer leurs caractéristiques, leurs ressemblances et leurs différences, dans l'espoir quelque peu illusoire que la linguistique réponde aux questions laissées en suspens par l'histoire. Entre le pédagogue et l'historien se déploie cependant un immense champ linguistique que la recherche peut sillonner : il ne s'agit plus seulement de parler avec des mots, mais aussi de faire parler les mots.

Caressant l'idée qu'on aurait une perspective inhabituelle sur le dialecte arabe hassāniyya de Mauritanie à travers l'examen d'une famille de mots, j'avais envisagé d'étudier la racine $B Y \delta o$ (cl. $B Y D$ ). C'est en effet à cette racine qu'appartient le terme $\partial l-B \bar{\imath} \delta$ ân qui est le nom que se donnent eux-mêmes les arabophones de Mauritanie (ceux que nous appelons les "Maures"). Par la suite trop de pistes se sont ouvertes pour que je n'essaie pas d'en suivre quelques-unes. On verra pour finir que les dialectes ont au moins des (petites) histoires à nous raconter.

\section{IL Y A BLANC ET BLANC}

La racine $B Y D$ est l'une des racines les mieux représentées dans l'ensemble du domaine arabe. Elle est attestée en effet dans tous les dialectes, mais avec des dif- 
férences formelles qui sont liées à la réalisation dialectale de la latérale vélarisée (phonème conventionnellement transcrit $d$ comme une emphatique simple). La forme $B Y D$ où $d$ est réalisée comme une vraie dentale emphatique, est fréquente, en particulier dans les dialectes de sédentaires qui ont perdu l'articulation interdentale (on la trouve du Maroc au Moyen-Orient en passant par Tlemcen, Tunis juif, Tripoli, le Tchad, l'Égypte, la Mecque, Aden, etc.). La forme $B Y \delta ̣$ est celle des parlers bédouins comme le hassāniyya (tant au Maghreb, au Machreq que dans la péninsule arabique), celle des dialectes sédentaires tunisiens et celle de quelques parlers citadins d'Algérie (Cherchell, Blida, Médéa...). Quant à la forme BYT, elle est limitée à quelques parlers de montagnards maghrébins où $d$ a tendance à passer à $t$ (cf. Ph. Marçais, 1956: 7 - 200 - 248; 1977: 9).

$\mathrm{Au}$ plan du contenu on retrouve également une forte cohésion puisqu'un même noyau sémantique apparait dans presque tous les parlers arabes. Ce noyau, qui se dégage assez clairement dans la plupart des unités lexicales de la racine, permet d'appréhender la valeur propre de celle-ci, même si on ne peut qu'imparfaitement en rendre compte avec des mots de la langue, sauf à souligner la présence récurrente du sème "blanc".

Dans ces conditions on comprendra que le lexème adjectival puisse apparaître comme premier, même si la présence du préfixe ' $a$ - aurait dû nous amener à chercher une autre base de dérivation, n'offrant par rapport aux consonnes radicales de la racine que l'ajout d'éléments vocaliques. En fait il semble assez normal que certains adjectifs soient traités sémantiquement comme des bases de dérivation, notamment les dénominations de couleur dans lesquelles la tradition a souvent voulu voir des mots "sémantiquement primitifs". En arabe, on peut en tout cas relever des faits morphologiques venant à l'appui de l'argument sémantique car les racines comportant des dénominations de couleur présentent une configuration dérivationnelle particulière à quatre éléments fondamentaux : d'une part le schème adjectival $a f^{\prime} a l$, d'autre par les schèmes verbaux II $f a^{\prime \prime} a l$, IX $f^{\prime} a l$ ou fa'all et X stafal.

\section{$1.1{\text { L'adjectif ebya } \hat{\delta}^{1}}^{1}$}

Il signifie "blanc" dans l'ensemble du monde arabe et fait partie de ces adjectifs de couleurs et de particularités physiques [abrégés dorénavant en : adjectifs de CPP] qui, en arabe classique, ont pour schème 'af'al et en hassāniyya, font af'al. La disparition du hamza initial n'a donc pas entraîné - comme dans les dialectes sédentaires maghrébins - la chute du préfixe vocalique. La forme féminine fait régulièrement beyọa (ou plutôt bề $\hat{y}^{\prime} a$ ), sur le schème fa' $l a$, tandis que le pluriel est $b \hat{\imath} \hat{o}$, avec assimilation de la voyelle par la semi-consonne (comme en arabe classique), alors que le schème régulier pour ces adjectifs est $f \sigma^{\prime} l^{2}$, cf. hom $h r$ pluriel de ahmar "rouge".

Contrairement à l'arabe littéraire, le dialecte maure admet pour les adjectifs en af'al l'existence d'élatif (comparatif-superlatif) de même forme, avec cette seule différence - caractéristique des dialectes - que le schème af'al d'intensité est inva-

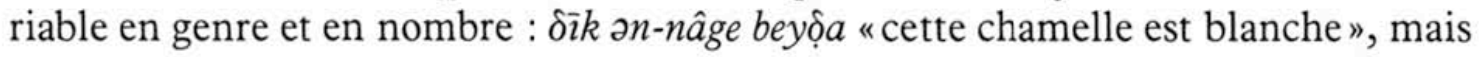
hiyye ebya ọ ən-nyâg "elle est la plus blanche des chamelles». En hassāniyya, les comparaisons suivantes sont passées à l'état d'expressions proverbiales : ebya ọ mon 


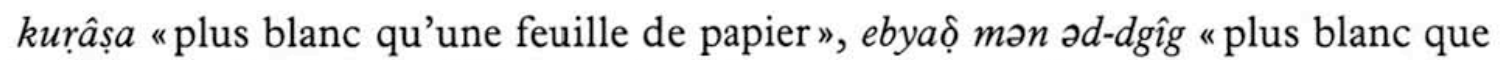
la farine", ebya ọ mən əš-šəgge "plus blanc que la percale".

L'existence d'une forme diminutive pour le schème $a f^{\prime} a l$ n'est pas, en soi, chose exceptionnelle puisqu'en arabe classique le schème 'ufay'il fournit un diminutif aux adjectifs de CPP. Le hass. afay'əl (ebeyyəọ "un peu blanc" comme diminutif de ebya $\delta$ "blanc») ne serait donc qu'un trait supplémentaire de conservatisme, propre aux dialectes bédouins ${ }^{3}$, s'il ne côtoyait pas régulièrement une autre forme diminutive afay al, celle de l'élatif, qui se distingue de la précédente uniquement par la variation de la troisième voyelle $(a \neq 2)^{4}$, (cf. C. Taine-Cheikh, 1988 a : 106).

\subsection{Le verbe beyya $\delta$}

La deuxième forme dérivée, à seconde radicale géminée 5 , a généralement une valeur dénominative dans les racines comportant un adjectif en af al de CPP, alors que les valeurs les plus fréquentes de II sont le causatif et l'intensif (ex. pour xbat "frapper" : xabbat "frapper plusieurs fois, faire frapper»). En hassāniyya, beyya signifie, comme en classique : "rendre blanc, blanchir, badigeonner de blanc". Il a aussi, mais moins fréquemment, le sens de "pondre" qui est très fréquent au Maghreb pour la forme nue.

Le collectif təbyâọ (qui signifie 1. "badigeonnage en blanc, blanchissement à la chaux"; 2. "fait de pondre, ponte") est le nom d'action régulier de beyyaọ, le dialecte maure préférant le schème $t \partial f^{\prime} \hat{a} l$ à celui de $t a f^{\prime} \hat{\imath} l$ comme schème de masdar pour II.

Le verbe passif ubeyya ọ (inacc. yubeyyaọ), qui dérive de beyyaọ par préfixation de $u$-, a le sens attendu de 1 . "être rendu blanc, être blanchi, être badigeonné de blanc, de chaux"; 2. "être pondu " et pour participe mubeyya $\delta$ (sur les formes passives en hassāniyya, cf. Taine-Cheikh, 1983). La différence formelle entre mbeyya et mubeyya $\delta$ est caractéristique de l'opposition entre participe actif (mbeyya $\delta$ 1. "blanchissant, badigeonnant de blanc, rendant blanc"; 2. "pondant") et participe passif (mubeyyaọ 1. "blanchi, badigeonné de blanc"; 2. "pondu»).

\subsection{Le verbe byâọ}

Dans l'ensemble du domaine arabe, il existe une autre forme verbale dénominative construite à partir de l'adjectif de CPP. Celle-ci, qui se distingue de la précédente par sa valeur intransitive de "devenir..." (byâọ yəbyâọ signifie "blanchir, devenir blanc"), n'a pas, au Maghreb, le même schème que dans les autres dialectes arabes (Égypte et Chypre compris, cf. A. Roth, 1975 : 75). En général la forme qui désigne une qualité physique en devenir est du type 'if alla et représente la $\mathrm{IX}^{\mathrm{e}}$ forme du classique, à la $3^{\mathrm{e}}$ radicale géminée. Dans tous les dialectes occidentaux, à notre connaissance, la forme usitée est du type $f^{\prime} \hat{a} l$, à voyelle longue après la seconde radicale. (On dit souvent que $f^{\prime} \hat{l} l$ pourrait venir de 'if âlla, la XI ${ }^{\mathrm{e}}$ forme du classique, celle-ci étant quasiment équivalente de la IX ${ }^{\mathrm{e}}$, cf. H. Fleisch, 1979 : 315-8).

Si ce schème semble bien caractéristique des dialectes maghrébins, ces derniers divergent cependant sur la conjugaison de l'accompli : les uns - dont le hassäniyya 
- abrègent la voyelle longue aux première et deuxième personnes par analogie avec les verbes à deuxième rad. glide (comme ' $\hat{a} d$ "il est devenu ", première pers. sg. ' $\partial d t$ ), tandis que les autres insèrent un suffixe -it par analogie avec les verbes à troisième rad. glide (comme šre "il a acheté", première pers. sg. šreyt) ${ }^{6}$.

\subsection{Le verbe stebya}

Bien que la $\mathrm{X}^{\mathrm{e}}$ forme ne soit pas limitée aux verbes dénominatifs comme la forme précédente, elle n'en fournit pas moins, pour beaucoup d'adjectifs de CPP, toute une série de verbes marquant le commencement d'un devenir. Ainsi stebya $\hat{\alpha}$ a-t-il comme premier sens celui de : "tendre vers le blanc, devenir blanchâtre, virer au blanc" (il signifie aussi "refuser qqc. par timidité - invitation, cadeau, repas, etc.", "être timide à l'excès").

\subsection{Les substantifs}

Le collectif beyọ est le terme courant en Mauitanie pour désigner les œufs, comme dans la majorité du monde arabe (en Tunisie, cependant, il a presque partout perdu ce sens et n'est guère employé que pour "testicules " ${ }^{7}$ ). Il y a donc homonymie entre le nom d'unité beyộ "un œuf " (duel beyọteyn, pluriel beyộat) et le féminin de l'adjectif "blanc».

Le substantif byâọ signifie "blancheur, (le) blanc de..." et intervient notamment dans quelques locutions désignant des parties du corps humain, cf. byâọ al-'ayn "le blanc de l'œil", byâo l-eyd "la paume de la main" (litt. "le blanc de la main"), byâ ${ }_{0} l o-k r a a^{\prime}$ "la plante du pied" (litt. "le blanc du pied").

Enfin nous avons le terme bị̀ân qui, en arabe classique, désigne "les blancs, les hommes de race blanche (opposé à nègres)". Il s'agit d'un collectif construit à l'aide du suffixe -ân qui est un élément essentiel en arabe dans la dérivation des ethniques (ou ethno-termes). Les suffixes -âni(yy) et -āniyye fournissent en hassāniyya des noms d'unité qui fonctionnent soit comme substantif soit comme adjectif.

Dans le dialecte maure, cependant, bīộn n'a pas la même extension qu'en classique. Le plus souvent, biộan désigne "toute personne parlant l'arabe (le dialecte arabe hassāniyya) comme langue maternelle". Il s'agit donc non plus d'une race, mais d'un groupe ethnique particulier se définissant culturellement par référence à sa langue : adéquation qui transparaît à travers l'appellation klâm al-bīôan (litt. "parler des Bīộn"), dont l'emploi est parfaitement synonyme de ceux de ḥassāniyya ou klâm hassân (litt. "parler des Hassân"). Ce glissement restrictif de la race à l'ethnie révèle d'un mouvement assez compréhensible d'égocentrisme (ou plus exactement d'ethnocentrisme), identique à celui qui, dans bon nombre de langues, nomme l'ethnie par le terme même de "humains" - on sait depuis les Grecs que les Autres sont toujours les "Barbares"...

L'entité maure se définit par une référence générale à un territoire (trâab al-bīọân "pays des Maures"), mais surtout culturellement, par référence à sa langue et sa musique, comme le traduit avec concision une belle expression proverbiale : ebeyọan mon tidinît woll Mânu (litt. "plus maure que la tidinit de wall Mânu" - la tidinit est l'instrument des hommes qui rappelle plus ou moins le luth ou le violon, et 
wall Mânu, le nom d'un célèbre griot), expression qui s'applique à quelqu'un qui parle avec finesse et clarté la langue maure, qui a assimilé la culture maure. La même idée se retrouve dans une autre expression signifiant également "il comprend la langue maure, la musique maure, la culture maure» : wəon-u beyọa (litt. "son oreille [est] blanche"). La tradition orale, loin d'occulter le lien étymologique entre abya ọ et bīộni, entre "blanc" et "maure (Blanc)", témoigne au contraire de la vitalité de ces rapports et joue avec toutes les ressources néologiques du dialecte (ebeyộan est le comparatif de bīọâni; sur l'élatif des quadrilatères en hassāniyya, cf. Taine-Cheikh, 1984).

\title{
2. A PROPOS DES NOIRS "BLANCS"
}

\section{1 es-Sūdân}

En arabe littéraire, al-bīdān "les blancs" s'opposent à al-sūdān "les noirs". Les deux termes désignent chacun un "caractère ethnique absolu, marque distinctive d'une race", mais, comme le montre bien Bernard Lewis, le blanc et le noir ont d'abord été des termes relatifs et personnels décrivant le teint d'individus particuliers (1982: 26-27) :

\begin{abstract}
"Pendant la période qui suivit immédiatement la mort du Prophète Mahomet, en 632 de notre ère, les grandes conquêtes islamiques portèrent la foi nouvelle à de vastes zones de l'Afrique et de l'Asie. Avec cette situation nouvelle, beaucoup de changements peuvent être observés dans la littérature de l'époque. D'abord, les termes décrivant la couleur des êtres humains furent moins nombreux, spécifiques ou spécialisés. Avec le temps, presque tous disparaissent à l'exception du noir, du rouge et du blanc qui prennent une coloration ethnique. Le noir désigne globalement les indigènes africains du Sud du Sahara, et leurs descendants. Le blanc, parfois le rouge (clair), désigne les Arabes, les Perses, les Grecs, les Turcs, les Slaves et les peuples vivant au nord et à l'est des terres habitées par les noirs. Parfois, pour les opposer aux Arabes et aux Perses, blancs, on attribue aux peuples du nord des qualificatifs signifiant albâtre, bleu pâle ou divers tons de rouge."
\end{abstract}

Tandis que les géographes et les historiens musulmans en viennent, petit à petit, à distinguer soigneusement les différents groupes de noirs africains (les Zanj, les Nūba, les Bujja...), le nom al-Sūdān reste l'appellation générale pour "Noirs ". Le terme de Bilād al-Sūdān "appliqué à toute la zone d'Afrique noire située au Sud du Sahara, du Nil à l'Atlantique, y compris les États noirs d'Afrique occidentale tels que le Ghana et le Songhay " (idem : 54), gardera toujours en arabe la signification très générale de "terre des Noirs". Ce n'est que par le détour des langues européennes ("traduction servile de la manière de s'exprimer des Européens" contre laquelle s'insurge le comte de Landberg) qu'on en viendra à dire fí al-Sūdān "au Soudan" comme si al-Sūdān désignait un pays.

Dans la plupart des parlers arabes, la racine $S W D$ est celle qui fournit l'adjectif "noir» et ses dérivés : aswad est communément utilisé partout à l'exception, semblet-il, du Maghreb occidental ${ }^{8}$ où on lui préfere la forme akhal.

En hassāniyya, comme dans les dialectes algériens et marocains, la racine $S W D$ n'est guère attestée en dehors du collectif sūdân et de son dérivé sūdâni(yy) dont le féminin est sūdāniyye ${ }^{9}$. Apparemment le terme os-südân a conservé le même sens que dans l'ensemble du monde arabe puisqu'il signifie "les Noirs", les hommes de race noire». En réalité son sens est beaucoup plus restreint qu'ailleurs car 
il ne s'applique guère qu'aux Noirs arabophones, c'est-à-dire aux Maures noirs, aux Bīôan de race noire, à l'exclusion des autres Noirs (en particulier des autres Mauritaniens de race noire: Toucouleurs et Peuls poularophones, Soninkés et Wolofs). La situation est assez complexe dans la mesure où biộan signifie soit l'ensemble des locuteurs hassāniyya, la totalité des personnes de culture maure, soit, restrictivement, les blancs, les Maures blancs par opposition aux Maures de race noire (esclaves et hruātîn ou esclaves libérés).

Cette appartenance / non-appartenance est compréhensible si l'on distingue bien les deux plans où se réalise le mécanisme d'opposition fondamentale ( il y a eux et nous", cf. R. Hoggart : 1970) commun à toutes les sociétés : d'une part, l'opposition eux $=$ les noirs / nous = les blancs, supportée (comme en classique) par l'opposition südân / biộân; d'autre part, l'opposition eux / nous pour laquelle " eux " n'a pas de terme unique et "nous" n'a pu s'exprimer que par déplacement-extension d'un des termes du niveau précédent.

En fait il y a deux mouvements indissociables qu'on ne saurait situer chronologiquement l'un par rapport à l'autre : un mouvement d'extension du champ réfentiel de biộan à tous les hassanophones, quelle que soit leur origine raciale, et un mouvement de réduction du champ réfentiel de südân aux seuls noirs arabophones.

\section{2 lo-ḥrāțîn}

Le terme de sūdân est fréquemment remplacé, pour désigner les Maures noirs, par celui de hrātîn (francisé en Haratin, sg. Hartani). Au sens strict, cependant, l'item hartâni (nom d'unité de hrạțîn, qui donne harțāniyye au féminin) indique un statut social, en même temps qu'une origine raciale : les hruâtîn sont des affranchis, des tributaires noirs, qui se distinguaient historiquement à la fois des serviteurs non libres ( $l a$ - $\left.^{\prime} b \hat{d} d\right)$ et des tributaires blancs (aznâge).

Si le sens de hrātîn est clair dans son usage actuel, celui de l'étymon soulève au moins autant de questions que l'origine historique des affranchis (cf. A. Leriche, 1951). Là où le mot hartani s'est révélé courant, c'est-à-dire principalement, outre la Mauritanie, dans l'Algérie méridionale de l'Ouest et au sud-est du Maroc $^{10}$, l'étymologie fournie par les lettrés est généralement celle de "libre au deuxième degré" (horr $\theta \bar{a} n i)$ qui aurait, selon Leriche, été donnée pour la première fois dans le Kitâb al 'istiqșa d'Ahmad b. Khālid al-Nâșiri al-Salāwī (idem : 25). Cette étymologie, fortement contestée par les linguistes européens, suscite le commentaire suivant de Ph. Marçais (1951: 12) : "découpage aussi enfantin que celui auquel on a pu soumettre le mot célibataire pour l'expliquer plaisamment par cœlum habitare "habiter le ciel». Tout aussi arbitraire lui apparaît le rapprochement de harțâni (réalisé $t$ ou $t$ selon les régions) avec la racine $H R \theta$ «labourer" pour des raisons à la fois de phonétique et de sémantique (le hartani fait de la petite culture à la houe dans les oasis, il est jardinier mais ne laboure pas).

Si les étymologies arabes sont peu convaincantes, la piste berbère pourrait être plus fructueuse. Il existe en effet un correspondant berbère de hartâni dans les confins sahariens (l'équivalence $d$ berbère $=t$ arabe étant tout à fait plausible) : āhardān chez les berbérophones du Tafilalet et du Moyen Atlas, hardanon chez les Aẓnâge de Mauritanie et achâdan en touareg.

Le terme harțâni pourrait donc représenter une arabisation du lexème berbère, 
d'autant que les attestations les plus anciennes jouent plutôt en faveur de l'antériorité de āhardān. En effet on a un témoignage de ce dernier dès le XII ${ }^{\mathrm{e}}$ siècle puisque G.S. Colin a relevé chez Ibn Khaldūn qu' "un prince almohade, le sayyid Abū Zayd, fils de sultan Yūsuf b. 'Abd al-Mu'min, portait l'épithète d'al-Harḍānī" (1975 : 237), alors que le premier enregistrement de hartani ne daterait, selon Marçais, que du XvirI siècle (1951: 11, note 2). La racine berbère, cependant, ne permet pas de résoudre véritablement le problème car elle ne donne que des noms d'une variété de lézard.

Si l'on en revient au sens de harțân dans les différents dialectes, on peut noter que les définitions rassemblées par Marçais tournent autour de trois idées principales : l'idée d'esclavage et d'affranchissement (ce sont des anciens esclaves, des descendants d'esclaves ou des esclaves affranchis); l'idée d'une couleur de peau ou d'une race noire; enfin l'idée de mélange, de métissage et de bâtardise.

Cette dernière idée de mélange (au sens de sangs mêlés, de mélange de races) me semble particulièrement intéressante à retenir car elle est sans doute la seule capable d'expliquer les autres emplois de la racine HRTNN. Ainsi en hassäniyya hartan signifie "se métisser (pour les chevaux de race uniquement)" et hartâni peut se dire du cheval de race croisée. Si harțan signifie également "devenir noir (pour le henné seulement, lorsqu'il laisse une couleur foncée sur la peau)", sans doute l'insistance est-elle sur le devenir, le passage du clair au foncé - et non sur l'aboutissement - car le principe mhartan qui dérive du verbe quadrilitère signifie explicitement "mélangé; de deux couleurs » et peut s'appliquer aussi bien à un animal qu'à une chose. Il semble possible de supposer, à partir de ces données, que la racine $H R T N$ a pour étymon "mélange de races" et plus spécifiquement, appliquée à l'homme, "métissage entre la race blanche et la race noire" ${ }^{11}$.

La traduction de "mulâtre», donnée par exemple par M. Beaussier, me semble bien correspondre à l'étymologie, même si elle apparaît mal adaptée à l'emploi actuel du terme hrāt țin. Si je me permets d'insister sur ce mot, c'est aussi parce qu'il constitue l'entrée française qui donne accès au lexème touareg achârdan "mulâtre (homme né d'un père blanc et d'une mère négresse, ou d'une mère blanche et d'un père nègre)" (Ch. de Foucauld, I : 134), alors qu'il existe un second mot pour désigner les haratin dans ce dialecte berbère : azeggar. Or ce lexème azeggar est de la même racine que ihouar qui signifie «être rouge (être de couleur rouge) - se dit de toutes les nuances ayant quelque chose de rouge, depuis le rose jusqu'au noir rougeâtre" (idem, II : 630-31). Du fait de l'étendue du spectre couvert par ce verbe, on comprend que, chez les gens de race blanche, ihouar puisse se dire des individus qui ont un teint rose coloré, et que chez les gens de race noire, par contre, ihouar s'applique au teint le plus clair.

Si l'on a pu relever, en deux points très éloignés de l'aire d'emploi de harțâni / āhardān, l'existence d'un synonyme (ou quasi-synonyme) ayant pour étymon une couleur - "noir" pour südân, "rouge" pour azeggar -, cela pourrait confirmer l'hypothèse que le terme concurrent harțâni / ăhardạn procède d'une autre logique dénominative et que la notation de couleur est absente de sa base étymologique. 


\section{DU NOIR ET DE LA NOIRCEUR}

Dans les dialectes où $a k h a l$ est devenu le terme général pour "noir ", la racine $K H L$ présente beaucoup de points communs avec les autres racines dont la base est un adjectif de CPP. Ainsi, en hassāniyya, retrouve-t-on pratiquement tous les schèmes présents dans la racine $B Y \delta \underline{~: ~}$

- l'adjectif $e k h a l$, son féminin kaḥle, son pluriel kəhl; l'élatif invariable $e k h a l$ (mən); les deux formes diminutives ekeyhal "un peu noir(âtre)" et ekeyhal (mon) "un peu plus noir (que)»;

- le verbe de deuxième forme kahhal "noircir, rendre noir", son passif $u k a h h a l$ et son nom d'action təkhâl;

- le verbe de IX forme khâl "noircir, devenir noir";

- le verbe de $\mathrm{X}^{\mathrm{e}}$ forme stekhal "virer au noir, tendre vers le noir, devenir noirâtre";

- le substantif khâl «noirceur".

Par ailleurs on a le mot $k \supset h l$ «antimoine " et le verbe dénominatif de cinquième forme qui en découle (tkahhal "se farder à l'antimoine"). Ce substantif se retrouve dans l'ensemble du monde arabe et est même passé en français comme nom de fard à paupière (le khôl). On imagine bien comment on a pu glisser, dans une partie du monde arabe (au Maghreb occidental), du sens de "collyre " à celui de "couleur noire", d'autant que certains lexiques, comme celui des Marazig, ont gardé trace de l'étape intermédiaire des "yeux noirs" (G. Boris, 1958: 523) :

a. kohl : "collyre";

b. tekhîl : "fait de mettre du collyre"; akhal : 1. "fardé au collyre";

c. akhal : 2. "qui a le tour des yeux noirs"; kohhêla : «loir aux yeux cernés de noir";

d. ${ }^{\circ}$ hîal: "bande noire sur la $\gamma^{a}$ rârạ".

En hassāniyya, l'adjectif ekhal a supplanté partout l'adjectif eswed dans le sens de "noir", à une exception près. En ce qui concerne la robe des animaux, en effet, on n'emploiera pas toujours la racine $K H L$ s'il s'agit de chameaux, de chevaux ou de bovins. Pour les chevaux et les bovins, on dira surtout edhem qui signifie "noirâtre, entre gris et noir (plus foncé que ešheb); bai foncé ". Pour les chameaux, le terme consacré est plutôt eswed; il a d'ailleurs donné naissance à des dérivés s'appliquant restrictivement à cet animal : swâd "devenir noir (chameau)", steswed "devenir noirâtre (chameau)", swâd "couleur noire (de la robe d'un chameau)". Les ovins-caprins sont donc les seuls gros animaux domestiques auxquels s'applique le qualificatif ekhal.

Il faut préciser par ailleurs qu'on n'utilisera guère l'adjectif ekhal pour qualifier une couleur de peau : on lui préfere celui de axọar. En fait le système qui permet de décrire le teint des personnes repose sur trois termes fondamentaux dont le sens varie en fonction de l'origine raciale :

- asfar (litt. "jaune») se dit des Blancs de teint clair;

- ahmar (litt. "rouge») se dit d'une part des Blancs bien mats, de teint cuivré, et d'autre part des Noirs de teint clair;

- axọar (litt. "vert-bleu") se dit d'une part des Blancs très mats, de teint très foncé, et d'autre part des Noirs de teint foncé.

Ekhal (noir) ne sera employé que pour donner l'idée de "noir franc, très foncé " par opposition à axọar; il sera donc à peine plus fréquent que ebya ọ ("blanc") 
qui ne s'entend guère puisque l'on pourra éventuellement avoir recours à l'adjectif az'ar (litt. "pâle") pour décrire une peau très blanche.

Si $e b y a \underline{\text { e t }} e k h a l$ sont plus ou moins exclus comme descriptifs de la pigmentation, il est intéressant de constater qu'ils fonctionnent fréquemment comme couple en opposition.

a. Byâo signifie "terrain où il y a peu d'arbres, terrain peu boisé " et trâab beyộa (litt. "terre blanche») "région de dunes, terrain aréneux, terrain sablonneux et découvert, région de sable" s'oppose à trâab kaḥle (litt. "terre noire") "pays boisé, terre argileux".

b. Ma'nâ-h ebya $\underline{0}$ "son sens est clair" s'oppose à ma'nâ-h ekhal "son sens est (délibérément) ambigu, il est à double sens".

c. Hžâb ebya ọ (litt. "talisman blanc") "talisman, amulette d'inspiration islamique, utilisant des formules tirées du Coran" s'oppose à ȟzâab ekhal (litt. " talisman noir») "amulette, médication de caractère magique, d'inspiration païenne".

d. Sur un plan moral, huwwe ebya $\delta$ "il est candide, de bonne foi, crédule, innocent ", s'oppose à huwwe ekhal «il est énergique, batailleur, prévoyant, dissimulé "; byâọ (ou byâọl-galb, litt. "blancheur du cœur ") "candeur, franchise " et byâọ əžžnân (sens litt. non connu) "crédulité, innoncence" s'opposent à khâl al-galb (litt. "noirceur du cœur") "cynisme, dissimulation" ${ }^{12}$.

e. En musique, les griots distinguent deux "voies" ou sous-modes pour chacun

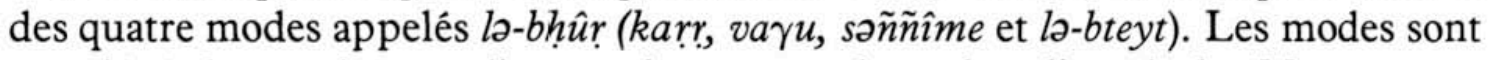
associés à des sentiments divers qui correspondent, dans l'esprit des Maures, aux sentiments éprouvés pendant les différentes époques de la vie : ils sont de la jeunesse (karr) à la vieillesse (lo-bteyt), en passant par l'âge viril (va $u$ ) et l'âge mûr (səñ̃n̂me). Certains griots dénombrent cinq modes au lieu de quatre, car ils parta-

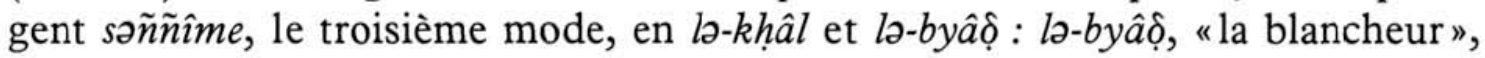
est associée à la douceur, à la nuance, à la délicatesse tandis que $l o-k h a ̂ l$, "la noirceur" est associé à la fierté et à l'énergie. Par ailleurs il y a opposition, à l'intérieur de chaque mode, entre la voie noire et la voie blanche (on dira khâl varu

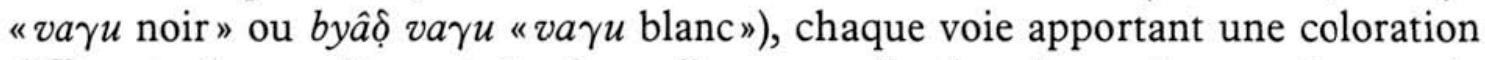
différente : la première entraîne les auditeurs vers l'enthousiasme alors que la seconde les pousse vers la douceur ou la tristesse (cf. M. Guignard, 1975).

Pour Leriche, la voie noire "se caractérise par des tons hauts et des tons bas; la voix qui peut être élevée subit des courbes très variées. C'est à elle que va la préférence des griots et elle est ainsi nommée parce que cette façon de chanter provient des Noirs" alors que la voie blanche a des "modulations harmonieuses, sans hausse de ton subite. D'origine arabe, c'est la plus ancienne des deux et c'est celle que préfere la bonne société maure" (1950: 730-1).

En ce qui concerne l'opposition entre beyt ekhal et beyt ebyaô, c'est-à-dire entre les mètres poétiques qui se chantent dans la voie noire et ceux qui se chantent dans la voie blanche, je crois avoir montré ailleurs (Taine-Cheikh, 1985) qu'il s'agit d'une opposition entre un rythme ascendant ou ïambique (avec "temps fort " sur la deuxième syllabe longue du pied : $u^{\prime}-$ ) et un rythme descendant ou trochaïque (avec «temps fort sur la syllabe longue du pied : $-u$ ). Il serait difficile de soutenir que le rythme ascendant (donc la voie noire dans laquelle ces mètres sont chantés) est moins arabe que le rythme descendant, car Fleisch a montré que non seulement la langue arabe elle-même a préféré les formes à rythme ascendant du type qatâl ou qitāl, aux formes à rythme descendant comme qātal et qātil, mais encore 
les mètres à rythme ascendant (dont le țawül, vers "héroïque" par excellence) sont plus fréquents que les autres dans la poésie en arabe classique (cf. Fleisch, 1968 : 63-7).

Sans vouloir nier par ailleurs l'influence probable de la musique négro-africaine sur la musique maure, je pense que l'assimilation byâ $\hat{c}=$ arabe, $k h \hat{a} l=$ négro-africain, s'intègre moins bien dans le schéma général de l'opposition blanc/noir que l'interprétation byâ $\hat{o}=$ douceur, $k h \hat{a} l=$ force.

f. Enfin l'on trouve assez fréquemment, dans l'histoire des tribus maures, la mention de deux fractions ou de deux sous-fractions rivales que l'on distingue par les épithètes de ebya $\delta$ et ekhal. Le cas le plus célèbre - mais il n'est pas unique est celui qui oppose $l d a w a^{\prime} l i$ blancs et ldawa'li noirs. L'idée la plus souvent émise est que le qualificatif de "noir" est dû à une ascendance maternelle noire ${ }^{13}$ : l'explication est même tellement fréquente qu'on ne peut manquer de se demander si elle n'est pas idéologique. Tout d'abord on remarquera, en ce qui concerne les $l d a w ' l i$, que $M$. Teffahi offre une autre explication qui, tout en étant plausible, a le mérite d'être en conformité avec le sens moral que nous avons vu précédemment : "Le schisme des Ida-Ouali et leur séparation en "blancs et noirs" s'explique par un différend surgi entre deux frères et leur oncle. L'un des deux frères s'étant montré arrogant avec lui, celui-ci attribua l'épithète de "noir " qui lui resta, tandis que l'autre qui lui donna des preuves de son respect reçut l'épithète de "blanc" (1953 : 16, note 20). Plus généralement on prendra garde au fait que le blanc et le noir pourraient bien fonctionner comme des indices de classement dualiste. Aucune des autres couleurs ne nous fournit autant d'exemples d'opposition dichotomique et le couple blanc/rouge est même le seul, me semble-t-il, à donner quelques expressions localisées de ce genre ${ }^{14}$; cf. :

- l-bahššrse l-beyọa (litt. "la gorge blanche"), "trachée artère", opposé à l-ḅəhššrse l-hamra (litt. "la gorge rouge»), "œsophage".

- šokwe beyộa (litt. "outre blanche"), "outre exclusivement réservée au lait baratté", opposé à šəkwe hamra (litt. "outre rouge»), "outre à lait de chamelle - pour conservation").

- kesb ebyaọ (litt. "troupeau blanc»), "troupeau composé d'ovins-caprins et de chameaux ", opposé à kesb ahmar (litt. "troupeau rouge»), "troupeau composé de bovins et d'ânes".

Derrière la paire élémentaire ebyaọ/ekhal (blanc/noir) - qui peut servir de support à l'expression d'une représentation duelle du monde - apparait donc une opposition secondaire ebyaơ/ahmar (blanc/rouge) qui indique peut-être la présence d'une forme triadique dont les organisations anthropologiques dites "dualistes" semblent rarement pouvoir se passer (cf. C. Lévi-Strauss, 1958 : 147-180).

Il est curieux de constater que j'aboutis, par des voies fort différentes, à des résultats assez proches de ceux trouvés par C. Lacoste-Dujardin dans son étude sur la taxinomie kabyle des couleurs :

"Une première paire contrastive domine l'ensemble des couleurs : «blanc/noir ", opposition élémentaire qui est celle de la clarté/obscurité dans la perception même de la couleur. Au sein de cette paire, chacun des termes parait monosémique, avec des valeurs symboliques simples et nettement opposées [...]. A côté de cette paire élémentaire les autres couleurs de base : "rouge ", "jaune" et "vert-bleu" sont à la fois moins précises dans leurs valeurs chromatiques, voire parfois équivoques, et plus polysémiques. [Mais] rouge est souvent introduit en tiers à côté du couple antagoniste blanc/noir pour constituer une trilogie qui tire sa force des effets contrastifs obtenus." (1985-6:151-2) 
On ne peut pas dire cependant que la trilogie blanc/noir/rouge (blancheur de la peau / noirceur des yeux et des cheveux / rouge des lèvres et des joues) qui alimente les portraits de l'aimée dans la poésie populaire maghrébine corresponde véritablement aux canons de beauté maure, car j'ai cherché en vain dans la poésie maure (lo- $\gamma n e)$ les poncifs en noir-blanc-rouge relevés par M. Virolle-Souibes. Il est vrai que le poète hassanophone s'attache plus aux défauts qu'aux qualités de sa maîtresse!... (cf. Taine-Cheikh, 1988b).

Par ailleurs je ne pense pas que ebya et ekhal soient porteurs, en hassāniyya, de valeurs aussi antithétiques qu'en kabyle où le noir est globalement négatif et le blanc globalement positif. Dans le dialecte maure, ekhal n'est pas vraiment associé à des connotations péjoratives. Il existe même un cas où c'est le rouge qui est nettement marqué comme négatif. Ne dit-on pas ḩəmr al-vūlāniyyîn (litt. "les rouges des tels") pour désigner "les moins nobles, les moins recommandables, les moins fréquentables des untels (de telle fraction...)"?

\section{OÙ L'ON RENCONTRE ENCORE DES NOIRS}

Jusqu'à présent, nous n'avons étudié qu'un ethnoterme désignant les Noirs, celui de os-Südân, mais il ne s'applique guère, comme nous l'avons vu, qu'aux Noirs arabophones, de culture maure. Les Négro-africains qui, en Mauritanie, sont des Noirs de langue poular, soninké ou wolof, sont appelés en hassāniyya: lo-kwâr. Ce nom collectif kwâr (dont le nom d'unité est, au masculin, kôri et, au féminin, köriyye) a pu avoir, selon les régions, des usages plus restrictifs visant à limiter l'emploi de ce lexème au groupe ethnique le plus proche. Mais en principe chaque communauté ethnique est désignée par un nom particulier : les Peuls sont appelés vüllân, les Toucouleurs tkārir, les Wolofs rōrmân/qōrmân et les Soninkés swânək ou sarqalle.

Quoi qu'il en soit, l'origine du mot kwâr pose un problème qui ne semble pas pouvoir se résoudre comme un emprunt à l'une des langues parlées par les Négroafricains de Mauritanie. Avant d'examiner les étymologies qui ont pu être avancées, je présenterai rapidement les divers lexèmes appartenant à la racine de kwâr.

Deux formes verbales dénominatives sont manifestement des néologismes créés à partir de kwâr :

- La première est une forme active à préfixe sa-(sekwar isekwar) dont la valeur est soit causative-factitive : "négro-africaniser, rendre semblable aux Noirs, faire adopter les coutumes des Négro-africains", soit déclarative : "traiter, considérer comme nègre".

- La seconde est une $\mathrm{X}^{\mathrm{e}}$ forme à valeur de réfléchi : stekwar yəstekwar, "se négroafricaniser, s'assimiler aux Noirs, adopter les coutumes des Négro-africains".

Comme $\mathrm{D}$. Cohen l'a montré, le verbe à préfixe $s a$ - (qui assume une grande partie des emplois de l'ancienne IV forme, du type 'af'ala) a des valeurs très voisines de celles du thème à seconde radicale géminée et il est probable que les formes en sa- ont été tirées des formes réfléchies en sta-, avec lesquelles elles sont en rapport d'actif à interne, par suppression du -t- (Cohen, $1963: 130-3)$. Cette transformation d'actif en réfléchi au moyen de l'affixe $t$ établit évidemment entre les deux formes verbales des rapports qui sont calqués sur les rapports qui existent 
entre les autres formes dérivées (II $/ \mathrm{V}^{\mathrm{e}}$; III ${ }^{\mathrm{e}} / \mathrm{VI}^{\mathrm{e}}$ et même $\mathrm{I}^{\mathrm{re}} / \mathrm{VII}^{\mathrm{e}}$ et $\mathrm{VIII}{ }^{\mathrm{e}}$; cf. TaineCheikh, $1983: 95 ; 1984: 288)$.

Deux questions restent cependant posées. La première, sur laquelle nous reviendrons plus tard, concerne le problème de l'influence éventuelle du berbère sur cette création analogique (on sait que dans certaines langues chamito-sémitiques - dont le berbère - la forme verbale à préfixe sa- est attestée, mais Cohen soulignait le fait qu'il n'avait pu relever aucune forme en $s a$ - qui soit empruntée au berbère).

La seconde concerne les rapports entre les deux formes causatives-factitives, la $\mathrm{II}^{\mathrm{e}}$ du type $\mathrm{fa}^{\text {e }}$ al et la pseudo-IVe du type saf'al. Il me semble en effet que la forme saf al n'est pas courante dans les racines d'origine arabe si la $\mathrm{II}^{\mathrm{e}}$ forme est bien attestée, sauf à avoir un sens tout à fait différent. Ainsi les verbes à préfixe sa-, que donne Cohen (sahmar, "rendre rouge, faire rougir de honte..." ; sakhal, "noircir", sabyaọ, "blanchir", etc., cf. 1963 : 131) ne sont-ils guère usités - d'après nos sources - lorsqu'ils sont dérivés d'adjectif de CPP et cela à la différence de verbes comme sekwar ou seslem. De même que seslem, "convertir à l'Islam ", se distingue de sellem, "saluer", sekwar s'oppose en effet, du moins chez certains locuteurs (et même s'il est généralement usité sous sa forme participiale) à kowwar, "avoir la forme d'une boule". A l'Est du pays on trouve encore, également de la racine $K W R$, un lexème kûra qui signifie "balle, ballon". Ce terme, qui est remplacé par towd au Sud-Ouest de la Mauritanie, vient de l'arabe littéraire kura, "sphère, globe, balle" ou küra (pl. kuwar), "district rural; petite ville; village; balle".

Pour M. Md Sâlem Ould 'Addoud (actuel ministre de la Culture et de l'Orientation islamique), qui est un grand savant dans les matières traditionnelles, le terme kwâr, "Noirs", vient de la racine arabe $K W R$ et spécialement de ce mot küra, "village", les Négro-africains étant avant tout des sédentaires par opposition aux éleveurs nomades d'origine arabo-berbère. Cette étymologie a cependant, me semblet-il, plusieurs inconvénients. Du point de vue de la forme, on n'a de correspondance exacte ni avec le singulier ( $k w a \hat{r} \neq k \bar{u} r a$ ) ni avec le pluriel (kwâr $\neq k u w a r$ ). Du point de vue du sens, le passage de "village" à "Noirs " est peu évident, même s'il n'est pas impossible. Enfin on s'attendrait, à défaut d'avoir ce sens en hassāniyya - ce qui est déjà un peu étonnant - à ce que küra soit un terme fréquent en arabe; or Ibn Manộur, citant Ibn Durayd, dit dans son Lisân al-'Arab que al-kūratu n'est pas considéré comme étant d'origine arabe (vol. 5 : 156).

Au total il me semble que, si jamais la racine classique avait fourni un ethnoterme en hassāniyya, ce ne serait pas kwâr, "Noirs", mais plutôt tkārîr, "Toucouleurs". En effet kāra ou kawwara, "rouler en spirale, par exemple un turban sur la tête", a peut-être donné en dialecte maure le verbe tekre (S.-O.) / tekker (E.), "mettre un morceau de tissu, enrouler un turban sur la tête pour porter des charges" (à rapprocher de tekrer, "faire et employer des tkārîr, des bottes de paille, pour les chameaux de transport"). Or, on peut imaginer que ces verbes, qui décrivent une technique de portage fréquente chez les Noirs, peuvent être formellement rapprochés du nom tekrûri (pl. tkärir) donné aux Toucouleurs. Je me garderai bien cependant d'affirmer quoi que ce soit dans ce domaine, en l'état présent de mes recherches, notamment avant une étude sérieuse du toponyme tekrûr qui est vraisemblablement de même origine que le mot tkārir ${ }^{15}$.

L'étymologie arabe de kwâr me paraissant peu convaincante, j'ai eu la chance, lors de mes recherches sur le mot harțâni, d'être amenée à suivre une piste touarè- 
gue qui s'est révélée fort intéressante. Me demandant ce que pouvait signifier le terme akaouar que Marçais donne entre parenthèses et sans explication, à la suite du tamacheq azeggarh, "rouge, de couleur foncée " qu'il signalait comme étant un usage équivalent de haratin (1951: 15), j'ai découvert que le verbe ikoual, en tamacheq, avait le sens de "être vert foncé» et, par extension, celui de "être foncé; être noir" : de n'importe quel ton de noir sauf celui de "noir franc" (Foucauld, 1952 : II, 835). De plus, il se trouve qu'en touareg toutes les couleurs fondamentales - et pas seulement le rouge - servent, comme en hassāniyya (et en arabe classique), à caractériser les différentes nuances de couleur de la peau :

"Les Kel Ah[aggar] classent les gens de race blanche, c.à.d. les Européens, les Arabes et les Touaregs, en 4 teints principaux : le blanc (imlal, "être blanc"), le rouge (ihouar, "être rouge"), le jaune (iroua, être jaune"), le brun (iroual, "être brun") [...]. Tous ces teints se trouvent chez les Kel Ah.; ceux qui dominent sont le blanc, le jaune et le brun, c.à.d. les teints mats. Les $\mathrm{Kel} \mathrm{Ah}$. classent les gens de race noire, c.à.d. les nègres, les mulâtres et les azeggar, en 3 teints principaux : le rouge (ihouar, "être rouge"), le vert foncé (ikoual, "être vert foncé" et p. ext. "être foncé; être noir"), le noir (oużżaf, "être noir"); le rouge comprend les teints des nègres, mulâtres et azeggar les plus clairs; le vert foncé comprend les teints plus foncés sans être tout à fait noirs; le noir comprend les teints qui sont noir franc." (Idem : 630-1).

On remarquera tout d'abord que le rapprochement entre $i k o u a l$ (de racine $K W L$ ) et $k w a \hat{r}$ (de racine $K W R$ ) ne doit pas soulever d'objection d'ordre phonologique car le passage de la liquide $l$ à la liquide $r$ est plausible.

Quant au rapport sémantique entre ikoual et kwâr, entre "noir de teint " et "de race noire " ${ }^{16}$, il est à peine besoin d'en souligner la filiation logique, puisqu'elle est strictement identique à celle qu'on a vue à l'œuvre précédemment dans les termes maures ol-biộan et əs-sūdân ainsi que dans le terme touareg azeggar.

Ce passage logique du nom de couleur à l'ethnoterme (au sens large) se retrouve même dans un autre terme du hassāniyya, terme dont nous n'avons pas encore parlé car il est d'un usage marginal et semble employé avec quelques fluctuations d'un individu à l'autre. Il s'agit de l'item $x ə \delta$ ri où l'on reconnâ̂t la racine $X \delta \hat{T} R$ qui a donné axọar, "vert, bleu; brun foncé, noirâtre (pour une pers. ou un animal)" : xəori n'est pas le seul mot de la racine où l'interdentale a perdu son emphase $(\hat{\gamma}>\delta)$ au voisinage de la voyelle $i$, car axaydər, "un peu vert..." (diminutif de l'adjectif au positif) s'oppose à axayọar r, "un peu plus vert...» (diminutif de l'élatif). Pour

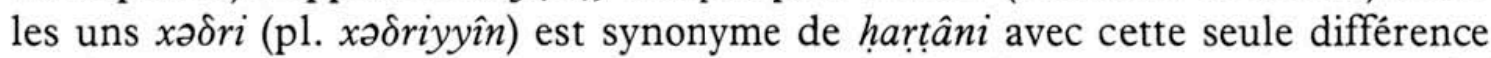
que la connotation du premier terme est plus neutre, alors que pour d'autres $x ə \delta r i$ peut se dire peu ou prou de tous les individus de peau noire, Négro-africains compris.

Avant de conclure, je voudrais revenir sur la racine $K W R$ et préciser deux points.

a. La racine $K W R$ nous fournit un exemple de forme dérivée en sa- (sekwar) dont la création pourrait avoir été influencée par le berbère puisque les noms de couleur ont également donné des formes verbales factitives en berbère et que celles-ci sont régulièrement à préfixe $s$-, cf. sekkouel, "rendre vert foncé», et sâkaouâl, "rendre hab. vert foncé" (Foucauld, 1952: II, 835).

Comme on pourrait à juste titre faire remarquer que les formes ont des sens différents, je donnerai le cas d'une autre racine. En hassāniyya la racine $N Y R$ comprend un certain nombre de mots qui ne sont pas d'origine arabe : senyer, "guide, faire le guide", astnâr / stenyer 5 , "trouver son chemin, bien savoir s'orienter", niyyar, "ayant un bon sens de l'orientation" et son comparatif enyer, àmnir / esenyâr, 
"guide", tānyîrət, "fait de savoir s'orienter". Il s'agit là encore d'une racine d'origine berbère puisque l'on a, par exemple en touareg (racine $N R$ ) : ener, "guider ", sener, "faire guider", emenir, "guide", tanāra, "fait de guider", etc. (Foucauld, 1952 : III, 1396,7).

Il semblerait que l'influence du berbère sur la création de la forme saf'al puisse donc être importante ${ }^{17}$, sans toutefois que le verbe dialectal doive nécessairement s'expliquer comme une généralisation à partir de l'emprunt d'une ou deux formes verbales isolées. Peut-être faut-il imaginer plutôt une influence diffuse, à travers les racines empruntées, de l'association d'une forme (à préfixe $s$-) et d'une valeur (factitive). L'une des principales divergences tient au fait que le saf'al arabe est souvent opposé à la $\mathrm{X}^{\mathrm{e}}$ forme (selon un raisonnement analogique propre au hassāniyya), au lieu de faire couple avec la première forme comme en berbère.

b. Comme on peut s'interroger sur l'ancienneté du mot kwâr, il n'est pas inintéressant de signaler que al-kôri est très ancien comme prénom puisque le célèbre al-Kôri wall Sîd al-Vâlli, des Owlâd Deymân, dont on situe la naissance en l'an mille de l'Hégire, se serait éteint en 1112 (1632).

En ce qui concerne les prénoms dans la société maure, je n'en ferai ici ni l'inventaire, ni l'historique - ce qui ne me permettra pas, par exemple, de préciser le sens qu'il faut donner aux prénoms (ol-)kôri et (ol-)kōriyye. Je me contenterai de signaler que les adjectifs de couleur, et spécialement ceux qui sont utilisés pour qualifier le teint des personnes, sont attestés comme prénoms. L-ebyaọ (litt. "le blanc"... ou "le candide" ?) et l-ekhal (litt. "le noir"... ou "le combatif"?) sont courants, mais l'on trouve aussi des diminutifs comme l-keyhal («le petit noir»), al-ḥaymar ("le petit rouge») et as-seyvar ("le petit jaune"), même si certains les deux derniers en particulier - ne sont guère donnés comme prénoms chez les Maures blancs.

\section{CONCLUSION}

Je pense que le lecteur qui s'interrogeait sur les caractéristiques du hassāniyya aura compris, au travers de l'étude approfondie de quelques racines, que le dialecte arabe parlé au Mauritanie présente un grand nombre de traits dialectaux propres aux dialectes maghrébins, nomades en particulier, à côté d'autres caractéristiques plus inhabituelles, qui font finalement du parler maure un dialecte original, à la fois conservateur et créatif.

En ce qui concerne les termes qui, en hassāniyya, désignent des ethnies, des groupes sociaux ou des races (et dans certains cas, les deux à la fois) - ce que j'ai appelé par commodité des "ethnotermes" -, on se gardera d'en tirer des conclusions générales sur les pourcentages respectifs d'arabe et de berbère dans le dialecte. On ferait en effet fausse route car on est plus proche en réalité de 10 ou $15 \%$ de berbère que de $50 \%$. On remarquera, par contre, que l'origine berbère (probable) de hria țîn et de kwâr pourrait témoigner de l'intrication très ancienne des populations blanches et noires dans cette partie de l'Afrique.

Par ailleurs il est intéressant de voir ce glissement réitéré de la couleur à l'ethnoterme - que ce soit en arabe classique, en hassāniyya ou en tamacheq - et la résistance de la langue pour éviter la confusion entre les deux (soit par l'adoption

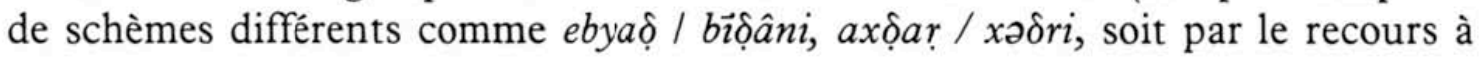


une autre racine comme $K H L / S W D$ ). Plus généralement, on aura noté que les adjectifs de couleur (qui appartiennent à des racines nominales ayant des caractéristiques relativement particulières) sont assez largement polysémiques, ce qui tend même à les rendre disponibles pour des emplois classificatoires.

I.M.R.S., novembre 1989

\section{Notes}

1. $[e]$ pour le phonème $|a|$ en contexte neutre.

2. Les dialectes arabes sont assez partagés sur ce schème du pluriel : au Maroc, une voyelle longue apparaît souvent (ex. bôyed à Tlemcen, bûyod à Rabat, buyed dans le Sous...) et dans certains parlers de sédentaires, $f u^{\prime} l$ tend à être remplacé par les pluriels à cumul $f u^{\prime}{ }^{\prime}$ inn et $f u^{\prime} l a ̄ n$ (cf. les dialectes de Palmyre, Mostaganem et Djidjelli ou les dialectes du Sud de l'Arabie méridionale). 3. Dans les dialectes de citadins et de villageois du Maghreb occidental, la disparition du schème 'ufay'il est partiellement compensée par une forme typiquement dialectale à redoublement (ex. à Tlemcen : bwî́böt, "blanchâtre", hmîmar, "rougeâtre").

4. Le cas de ebeyya ô mon est un peu spécial car il est moins spontanément employé dans le sens du diminutif "un peu plus blanc que" que dans celui de "blanchissant mieux que». En effet ebeyyaọ est aussi le comparatif de mbeyyaọ, "blanchissant" (sur l'élatif des verbes dérivés, cf. Taine-Cheikh, 1984).

5. Sur la dérivation des formes verbales dans les racines à deuxième radicale glide, cf. TaineCheikh, 1987.

6. L'abrègement est plus fréquent en Tunisie et dans les dialectes bédouins tandis que les dialectes citadins et ruraux du Maroc et de l'Oranie préferent la conjugaison sans abrègement ou à suffixe -ît (cf. Ph. Marçais, $1977: 64-65)$, mais il existe de nombreuses exceptions à la règle puisque, par exemple, Djidjelli et Cherchell "abrègent" alors que le dialecte des Ulâd Brāhîm de Saïda "suffixe".

7. En général, comme en classique, bayd cumule les deux sens de "œufs" et "testicules", mais en hassāniyya, dans le second cas, on emploiera plutôt le terme byấọ.

8. On trouve aswad, non seulement en Égypte et à Kormatiki (Chypre), mais aussi au Tchad et en Libye (Tripoli). La zone charnière pourrait être la Tunisie et l'Est algérien, car aswad apparaît à Djidjelli à côté de akhal mais est donné à Takrouna comme un terme emprunté, moins courant que l'autre (W. Marçais, 1959 : 1926).

9. Sur l'emploi de eswed (et de ses dérivés) appliqué à la robe du chameau, cf. ci-dessous.

10. "Chez les Chaâmba, à Ouargla, El-Goléa, In-Salah, Timimoun, Adrar, Beni-Ounif, ColombBéchar, Abadla, Beni-Abbès, Tabelbala, Tindouf, en Mauritanie, dans le Sous, le Dra, au Tafilalet et dans l'Atlas marocain" (Ph. Marçais, 1951: 11).

11. Les connotations d'un tel mélange sont telles qu'elles peuvent expliquer les autres usages maghrébins de la racine $H R T N$ (tels que "s'abâtardir" ou "s'acclimater " pour le verbe; "terre non franche" ou "sauvageon ou arbre non greffé" pour le substantif).

12. Les verbes de la racine $K H L$ sont également employés dans un sens moral : kahhal m'a hadd, "se comporter de façon réservée, calculatrice, cynique avec quelqu'un"; khâl, "être peu pénétrable, calculateur, dissimulé, discret".

13. Il est assez remarquable à cet égard qu'en attribuant une mère mélanoderme aux "noirs", on leur octroie un trait qui, du point de vue primordial, est neutre puisque la généalogie, pour l'essentiel, ne prend en compte que la filiation paternelle.

14. On trouvera d'autres locutions construites avec ebya $\delta$ - mais sans opposition dichotomique - dans Taine-Cheikh, 1988a : 171-2.

15. Il est tout à fait étonnant par exemple que, dans les ouvrages de lettrés maures, les appellations Bilād al-Takrūr et Bilād Sinqüt puissent être parfaitement équivalentes (cf. Ould Cheikh, 1985 : 349 , note 96$)$.

16. On signalera à toute fin utile ce qui n'est sans doute que le fruit du hasard - mais peut paraitre comme une étrange survivance du sens premier de ikoual ("être vert") : les noms des étoiles al-kubriyât ou əl-kôri w-əl-bíọâni dont l'apparition alterne dans l'année. L'une, verte (əlkôri), est visible en saison froide tandis que l'autre, rougeâtre (ol-biôâni), est visible en saison 
d'hivernage. L'explication rationalisante qui nous a été donnée est la suivante : la saison froide est la bonne saison pour les Noirs alors que l'hivernage est la bonne saison pour les pâturages - donc pour les Maures.

17. Il y aurait certainement d'autres formes dialectales en saf $a l$ à examiner de près (je pense par exemple à segre, "excuser", et sedbe, "faire partir l'après-midi»).

\section{Bibliographie}

Beaussier (M.), 1958, Dictionnaire arabe-français, Alger, 1093 p.

Boris (G.), 1958, Le parler arabe des Marazig, Klincksieck, Paris, 686 p.

Cohen (D.), 1963, Le dialecte arabe hassāniyya de Mauritanie, Klincksieck, Paris, 292 p.

Colin (G.S.), 1975, "Article Harțānỉ", Encyclopédie de l'Islam, tome III, Brill/Maisonneuve, Leyde/Paris, 237-8.

FleIsCH (H.), 1968, L'arabe classique - Esquisse d'une structure linguistique, Beyrouth, $271 \mathrm{p}$. FleisCH (H.), 1979, Traité de philologie arabe, vol. II, Beyrouth, 626 p.

Foucauld (père Ch. de), 1951-52, Dictionnaire touareg-français, 4 vol., Impr. Nat. de France, Paris. Guignard (M.), 1975, Musique, honneur et plaisir au Sahara, Geuthner, Paris.

HogGart (R.), 1970, La culture du pauvre, Éd. de Minuit, Paris, 420 p.

Lacoste-Dujardin (C.), 1985-6, "Du génie rouge à la femme blanche... et noire", L.O.A.B., 16-17, Paris, 135-155.

LERICHE (A.), 1950, "Poésie et musique maures", Bull. I.F.A.N., t. XII, 710-50.

Leriche (A.), 1951, "Les Haratin (Mauritanie)", Bull. de liaison saharienne n 4, 24-29.

Levi-Strauss (Cl.), 1958, Anthropologie structurale, Plon, Paris, 452 p.

Lewis (B.), 1982, Race et couleur en pays d'Islam, Payot, Paris, 163 p.

Manzūir (Ibn), s.d., Lisān al'-Arab, Dar Sader, Beyrouth, 15 vol.

MarÇaIs (Ph.), 1951, "Note sur le mot Hartani", Bull.de liaison saharienne, n 3, 10-15.

Marçais (Ph.), 1956, Le parler arabe de Djidjelli, Maisonneuve, Paris, 648 p.

MarÇAIS (Ph.), 1977, Esquisse grammaticale de l'arabe maghrébin, Maisonneuve, Paris, $284 \mathrm{p}$.

Ould CHeIkн (A.W.), 1985, Nomadisme, islam et pouvoir politique dans la société maure précoloniale (XIe s.-XIX ${ }^{e}$ s.), doctorat sous la dir. de L.V. Thomas, Université de Paris V, 3 vol., $1010 \mathrm{p}$.

Roth (A.), 1975, Le verbe dans le parler arabe de Kormatiki (Chypre), Geuthner, Paris, 118 p.

TAINe-Cheikh (C.), 1983, "Le passif en hassāniyya", Matériaux arabes et sudarabiques (G.E.L.L.A.S.) n 1, Geuthner, Paris, 61-104.

Taine-Cheikh (C.), 1984, "Un exemple de créativité lexicale : l'élatif en hassāniyya", Arabica, t. XXXI, fasc. 3, 274-305.

TAINe-Cheikh (C.), 1985, "Le pilier et la corde : recherches sur la poésie maure", Bull. of S.O.A.S., vol. XLVIII, Part 3, Londres, 516-35.

TAINE-Cheikh (C.), 1987, "Sous-classes verbales et racines "anormales" en hassāniyya ", Matériaux arabes et sudarabiques, $\mathrm{n}^{\circ}$, Paris, 53-93.

TAINE-CheikH (C.), 1988a, Dictionnaire hassäniyya-français, vol. 1, Geuthner, Paris, CIII +177 p.

Taine-Cheikh (C.), 1988b, "Les diminutifs dans le dialecte arabe de Mauritanie", Al Wasît (Bull. de 1'I.M.R.S.), $\mathrm{n}^{\circ}$ 2, Nouakchott, 89-118.

Teffahi (M.), 1953, El Wasit par Ahmed Lamine Ech Chenguiti, I.F.A.N., Saint-Louis, 150 p.

Virolle-Souibes (M.), 1985-6, "Les couleurs de l'amour ou l'art de cultiver les poncifs", L.O.A.B., 16-17, Paris, 101-134. 